\title{
Behavior of Hydrogen and Nitrogen in Tungsten, as Divertor Wall of a Fusion Reactor
}

\author{
Sergio Beneitez ${ }^{1}$, Juana L. Gervasoni ${ }^{1,2}$ and Juan Carlos Furnari ${ }^{3}$ \\ 1. Bariloche Atomic Center, CNEA (National Atomic Energy Commission), Bariloche 8400, Argentina \\ 2. CONICET (National Council of Scientific and Technical Research), Argentina \\ 3. Ezeiza Atomic Center, CNEA (National Atomic Energy Commission), Ezeiza 1802, Argentina
}

\begin{abstract}
The analysis of the interaction of hydrogen, nitrogen (and their isotopes) with tungsten is important, since this material is a strong candidate to form the first wall of fusion reactors for both magnetic and inertial confinement, and these atoms have a very sensitive (desired and unwanted) interaction with it. For this purpose, we study the effects and electronic state densities of atomic hydrogen and nitrogen in pure tungsten, in order to analyze some important properties such as the density of states of the system. Focusing on this application, this work is a preliminary study of the behavior of atoms of hydrogen and nitrogen, on a surface of tungsten on the three sites of the cell: top, hollow and bridge. We use a program simulation based on the DFT (density functional theory) implemented in the Open-Source Code Quantum Espresso, in order to obtain the adsorption energy and the density of states of the systems.
\end{abstract}

Key words: Tungsten, electronic properties, fusion.

\section{Introduction}

The new reactor concepts, including fusion, are characterized by greater efficiency, better utilization of nuclear fuel and waste minimization. To achieve high performance parameters, ongoing research and testing of new materials is necessary [1].

The recent conceptual designs of a fusion reactor, both confinements as magnetic inertial [2] consider tungsten (W) as one of the functional materials [3-5], candidate to be part of several engineering units, in particular the first wall or first containment barrier, the divertor $[5,6]$, subjected to extreme conditions, temperatures and irradiation, even higher than those in a fission reactor in terms of fluxes, spectrum hardness and number of source terms (neutrons, gamma, $\mathrm{H}, \mathrm{H}_{2}$, ${ }^{4} \mathrm{He},{ }^{3} \mathrm{He}$ ). Of all metals and alloys, tungsten has the highest melting and boiling point (PF-3,422 ${ }^{\circ} \mathrm{C}$, PE-5,930 ${ }^{\circ} \mathrm{C}$ ), exhibits high hardness and good heat

Corresponding author: Juana L. Gervasoni, Ph.D., research fields: metallic and nanostructured, of applied research management. capacity at high temperature $\left(11.3 \mathrm{~kW} / \mathrm{m}\right.$ at $\left.1,000{ }^{\circ} \mathrm{C}\right)$, is characterized by a high thermal conductivity and a low coefficient of thermal expansion, so that it presents a good resistance to thermal shock. Tungsten has a high threshold for physical spray energy, so it is a low erosion material when irradiated with different types of particles; has a low solubility of $\mathrm{H}_{2}$, low retention of tritium and its activation is low [1]. The conditions in which the fusion is conceived prioritize the study of the damage on tungsten caused by hydrogen and its isotopes under extreme conditions (pressure, temperature). However, relevant experimental results were obtained for mechanisms of formation of molecules of $\mathrm{NH}_{3}$ on tungsten surface [7], due to the interaction of it with $\mathrm{H}, \mathrm{N}, \mathrm{H}_{2}, \mathrm{~N}_{2}$ particles of the plasma at low temperatures [8]. Taken this into account, we will analyze the mechanisms of adsorption of $\mathrm{H}, \mathrm{H}_{2}, \mathrm{~N}, \mathrm{~N}_{2}$ as precursors of the formation of $\mathrm{NH}_{3}$ in tungsten. The term adsorption is introduced in Ref. [9] to connote the condensation of gases on surfaces, produced by the forces of interaction between the solid and the molecules or 
atoms of the gas. Basically these forces are of two kinds: physical, with predominant interactions of Van der Waals type, and chemical interactions that have taken into account the chemical bonds of the system. Thus, adsorbate is defined as the unit (atom or molecule) adsorbed by the metal surface.

\section{Materials and Methods}

In this article we employ the DFT (functional density theory) [10-14] implemented in the Open-Source Code Quantum Espresso [15]. In order to calculate the adsorption energy of the system, we solve the Kohn-Sham equations in the frame of the GGA (generalized gradient approximation), using the PBE (Perdew-Burke-Ernzerhof) functional [13] with ultrasoft Vanderbilt pseudopotential. These equations were solved in a self-consistent way, for a periodic system of symmetry of translation in 3D for the case of the calculation of the bulk. A slab was defined for calculations with 2D symmetry with the extended third dimension (in the $\mathrm{z}$ direction), in order to simulate a surface by increasing the distance between cells.

The Kohn-Sham orbitals are expanded in plane waves and the cut-off value for the energy is $650 \mathrm{eV}$. The geometric optimization is performed using the BFGS (Broyden-Fletcher-Goldfarb-Shanno) algorithm. The adsorption energy is defined as [9]:
$E_{\text {ads }}=-\left(E_{\text {molecule/surface }}-E_{\text {surface }}-E_{\text {molecule }}\right)(1)$ where $E_{\text {molecule/surface }}$ is the energy of the surface-molecule (or atom) system, $E_{\text {surface }}$ is the energy of the surface of the adsorbate and $E_{\text {molecule }}$ is the energy of the isolated molecule (or atom). According to this definition, positive adsorption energy corresponds to an attractive interaction between the adsorbate and the surface. The dimensions of the super cell were defined by parameterizing the increase of the corresponding dimension considering the variation of the adsorption energy $E_{a d s}$, taking as a criterion a variation of less than $1 \%$ of it. The cutoff and the mesh of the first Brillouin zone were determined in the same way. We define ecutwfe and ecutrho as the energy cutoff (Ry) and the kinetic energy cutoff for charge density and potential. We selected for this system: ecutwfc $=30$, ecutrho $=10 *$ ecutwfc. The size of the k-mesh is $10 \times 10 \times 1$. After the relaxation of the parameters to determine $E_{a d s}$, and the subsequent selfconsistent calculation, the calculations of the DOS (density of states) were implemented.

\section{Results}

Considering $\mathrm{E}_{\mathrm{ads}}$ as the parameter to be stabilized, we take a slab of 10 atomic layers in the direction (001) with a lattice of 74 atoms. The same size is taken in the perpendicular directions (010), and (100), as shown in Fig. 1. In each of the three crystallographic

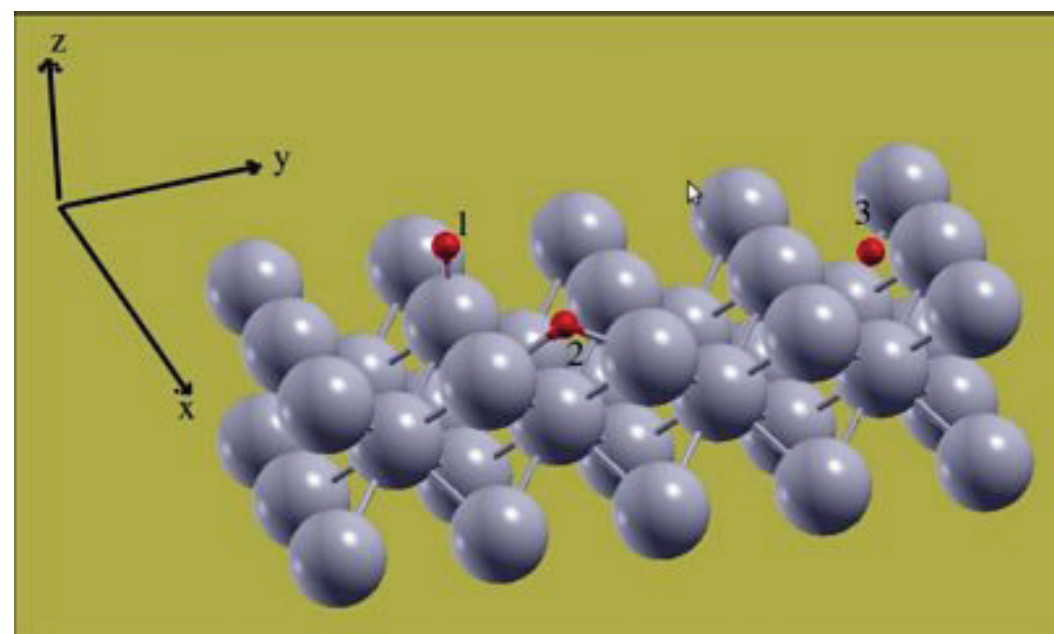

Fig. 1 Scheme of the considered system W-atom: (1) top interaction site, (2) bridge interaction site, (3) hollow interaction sites. 
Table 1 Energy of adsorption for the tree tipes if interaction sites, for $\mathrm{H}$ and $\mathrm{N}$, measured in $\mathrm{eV}$.

\begin{tabular}{llll}
\hline Atom & Top & Hollow & Bridge \\
\hline $\mathrm{H}$ & 3.630487 & 3.645677 & 4.729204 \\
$\mathrm{~N}$ & 8.919015 & 11.51710 & 10.69447 \\
\hline
\end{tabular}

Table 2 Distances W-atom in A.

\begin{tabular}{llll}
\hline Atom & Top & Hollow & Bridge \\
\hline $\mathrm{d}(\mathrm{W}-\mathrm{H})[\mathrm{A}]$ & 1.773792 & 2.205204 & 1.918391 \\
$\mathrm{~d}(\mathrm{~W}-\mathrm{N})[\mathrm{A}]$ & 1.72925 & 1.849850 & 1.894001 \\
\hline
\end{tabular}

directions of tungsten, we study different sites and angular dependence: Top: the atom interacting directly with an atom of the surface (Fig. 1(1)). Hollow: the atom interacts with 4 atoms of the surface (Fig. 1(2)). Bridge: The atom interacting with two atoms on the surface (Fig. 1(3)). The atoms were fixed at a distance between $\mathrm{d}$ atom- $\mathrm{W}=2 \AA$, with respect to the surface. The positions were allowed to fully relax. The network parameter for the optimized system, $\mathrm{a}=$ $3.162 \AA$, diverging from the reference value $\mathrm{a}=3.164$ $\AA$ obtained from Ref. [16], in less than $0.1 \%$, was used in the calculations.

Table 1 shows the adsorption energy of the hydrogen and nitrogen atoms, in both it was observed that the energy of adsorption does not vary appreciably according to the site of interaction. Table 2 lists the distances resulting from the relaxation calculation, between the adsorbated atoms and metal surface atoms $\left(\mathrm{d}_{\mathrm{atom}}-\mathrm{W}\right)$.

\subsection{Density of States}

\subsubsection{Hydrogen on $\mathrm{W}$}

In this sub section we show the DOS of $\mathrm{H}$ on $\mathrm{W}$, for the three sites: hollow, bridge and top.

Let us remember that the DOS of a system describes the number of states per interval of energy (at each energy level) available to be occupied. PDOS (projected density of states) is related with DOS and is the number of available states for electrons to occupy projected on atomic orbitals. It means that it is possible to see which shell $(s, p, d, f)$ and orbital for specified atom has the biggest share in DOS.
Note that for the following graphics, $\mathrm{E}_{\mathrm{Fermi}}$ for the pure $\mathrm{W}$ is shown in green, and for the $\mathrm{WH}$ system is shown in red.

In Fig. 2a we show DOS on W, and W-H system for bridge site: it can be noticed that near the Fermi level, the W-H system continues to be conductive although to a lower extent with respect to the $\mathrm{W}$ (pure) system. For the projected state density (Fig. 2b) it can be noted that near the Fermi level the $3 \mathrm{~d}$ orbital is the one that contributes the most to the transport properties or to the conductive character: hydrogen does not contribute.

For $\mathrm{H}$ on hollow site: we see in Fig. 3a that for this interaction site it can be noticed that near the Fermi level the density of the state does not change, so the system's characteristics do not change. In Fig. 3a, we see that, as for the bridge interaction site, the projected state density near the Fermi level, the $3 \mathrm{~d}$ orbital gives the principal contribution to the transport properties or to the conductive character. In this case, hydrogen does not change the behavior of DOS.

Finally, in Fig. 4a we show the properties for top site: just as for the bridge and hollow sites, for this interaction site it may be noted that near the Fermi level, the density of the state does not change, so the system keeps its driver's property unalterable or does not change.

On the other hand, from Fig. $4 \mathrm{~b}$ it is observed that near the Fermi level for this site, it is the orbital s of the hydrogen atom that governs or contributes slightly to the conductive character.

\subsubsection{Nitrogen on $\mathrm{W}$}

In this subsection we show the DOS of N on $\mathrm{W}$, for the three sites: hollow, bridge and top, following the scheme of the precedent subsection.

In Fig. 5a we show DOS on W, and W-N system for bridge site: it may be noted that the Fermi level of the W-N system shifts slightly for higher frequencies (or energies) and decreases near the $\mathrm{E}_{\text {Fermi. }}$.

For the PDOS, from Fig. $5 \mathrm{~b}$ it can be noted that the orbital $2 p$ of the tungsten competes with the nitrogen 


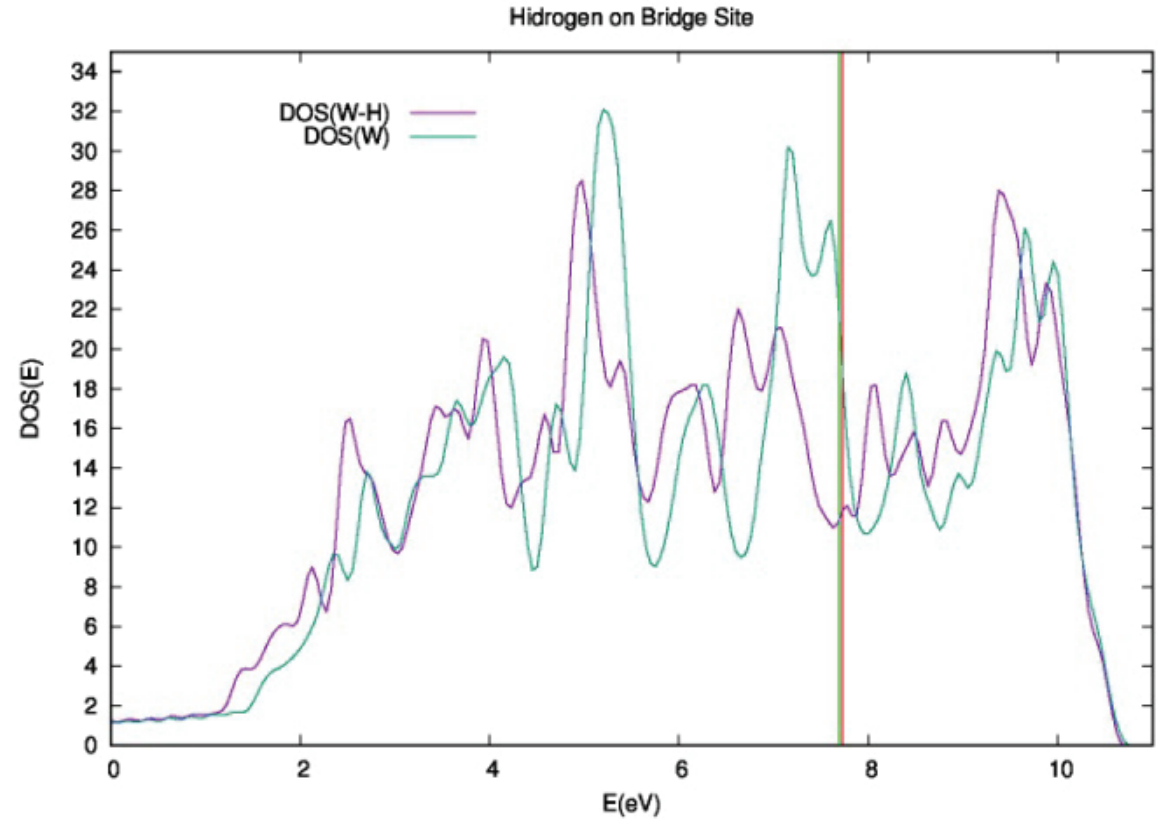

(a)

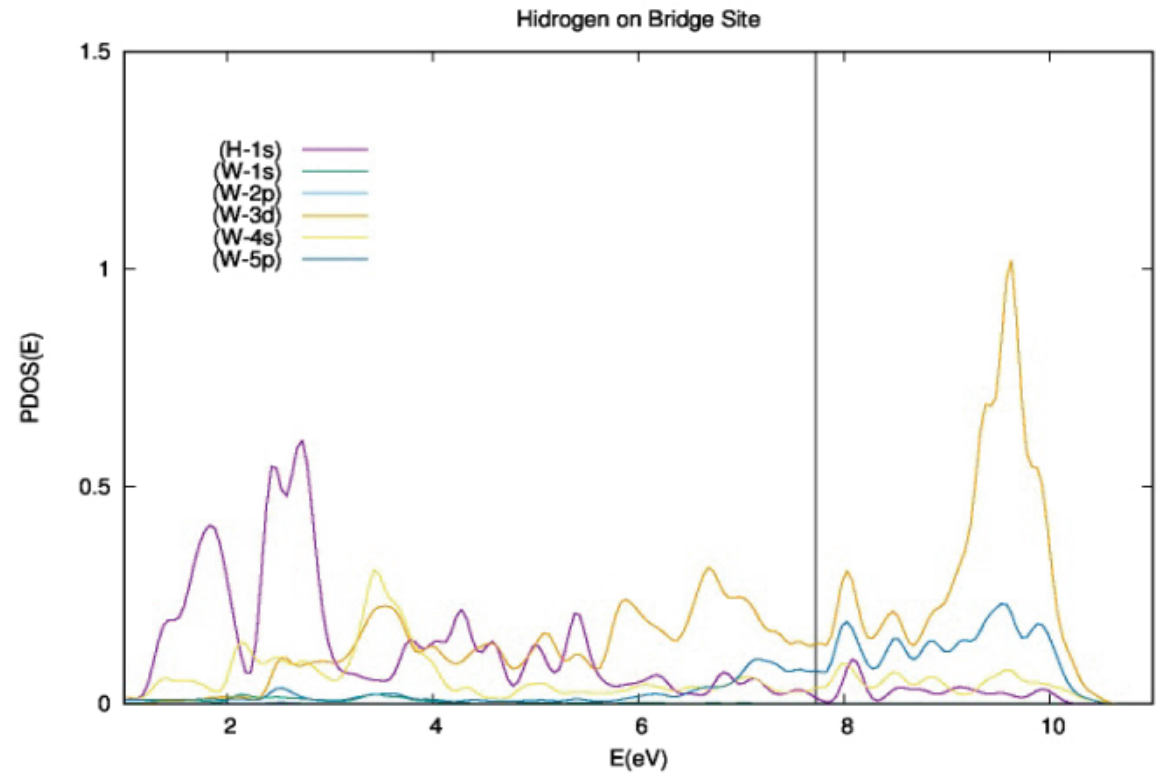

(b)

Fig. 2 (a) DOS of H-W on bridge site; (b) PDOS (projected DOS) of the same system. 


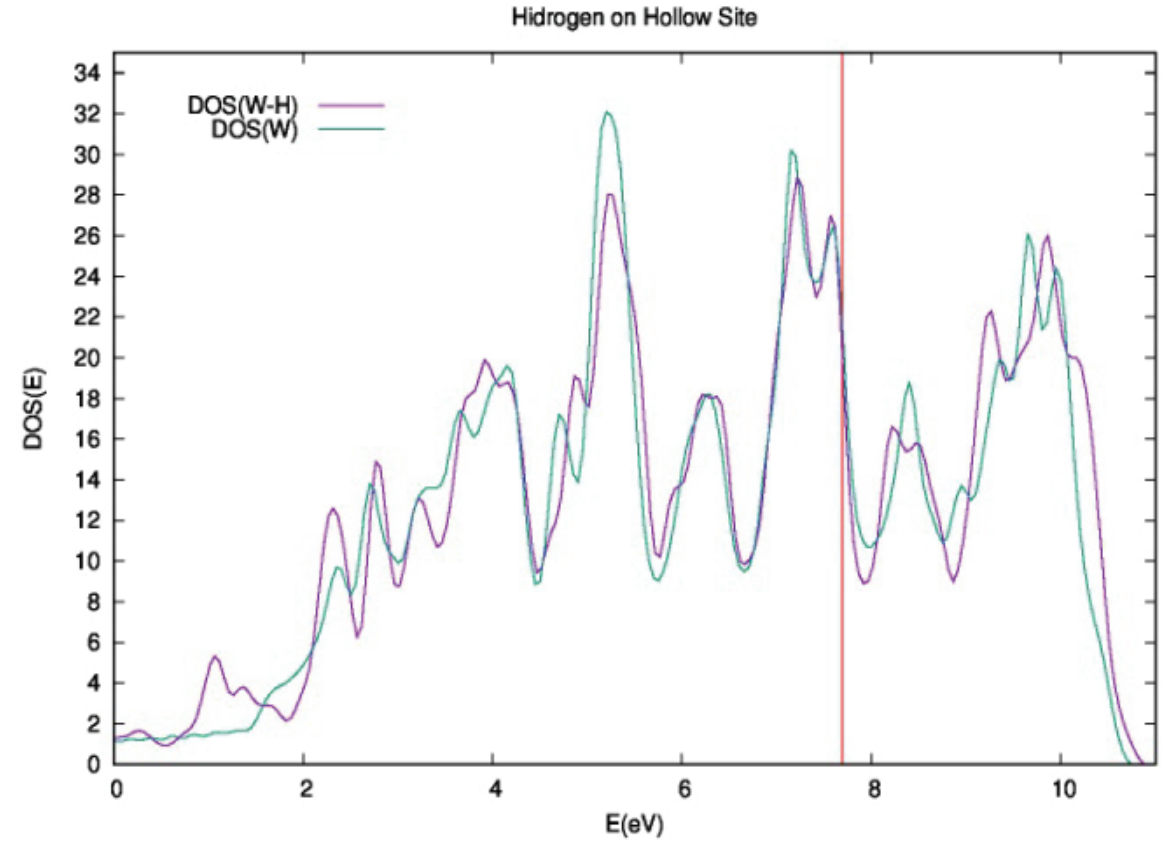

(a)

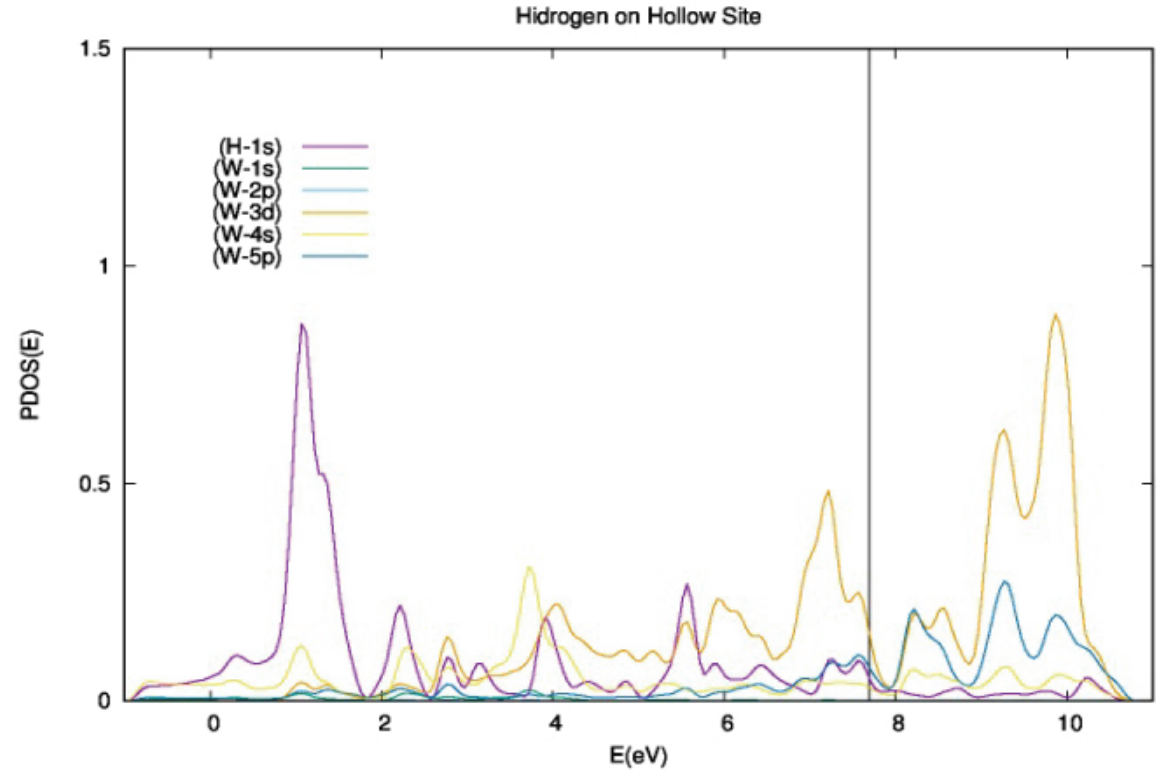

(b)

Fig. 3 (a) DOS of H-W on hollow site; (b) PDOS of the same system. 


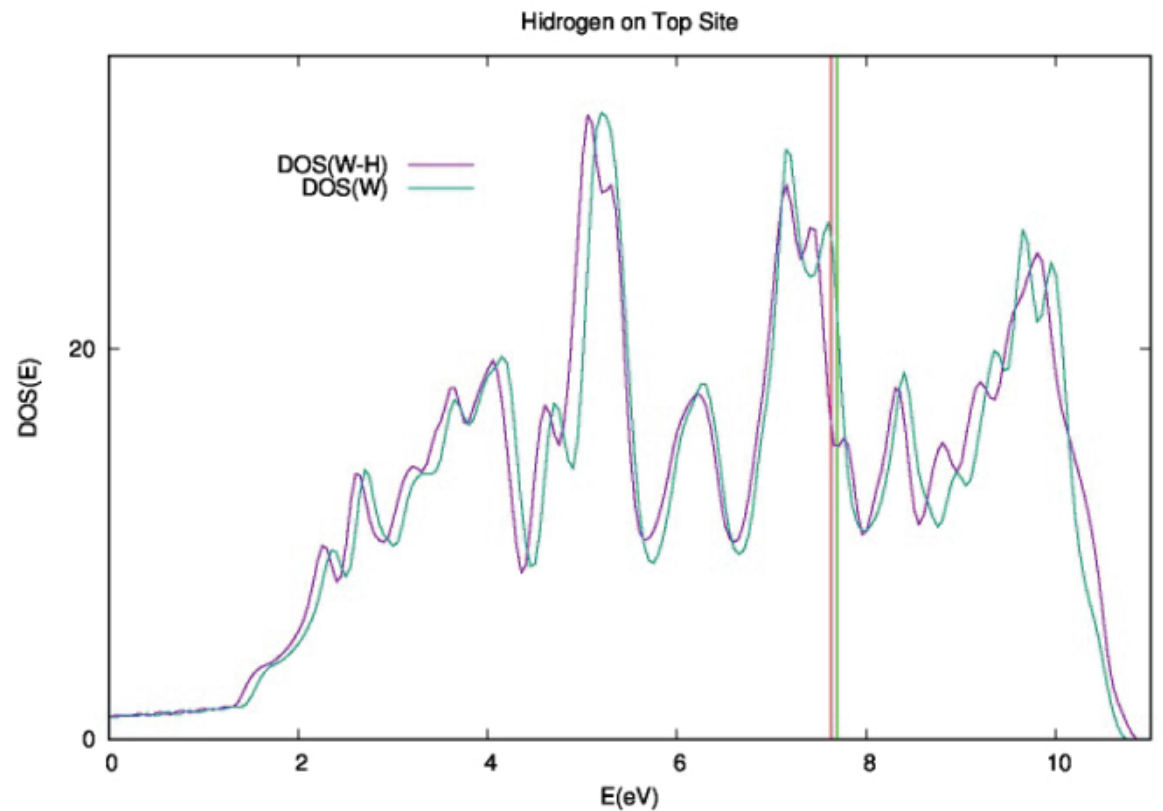

(a)

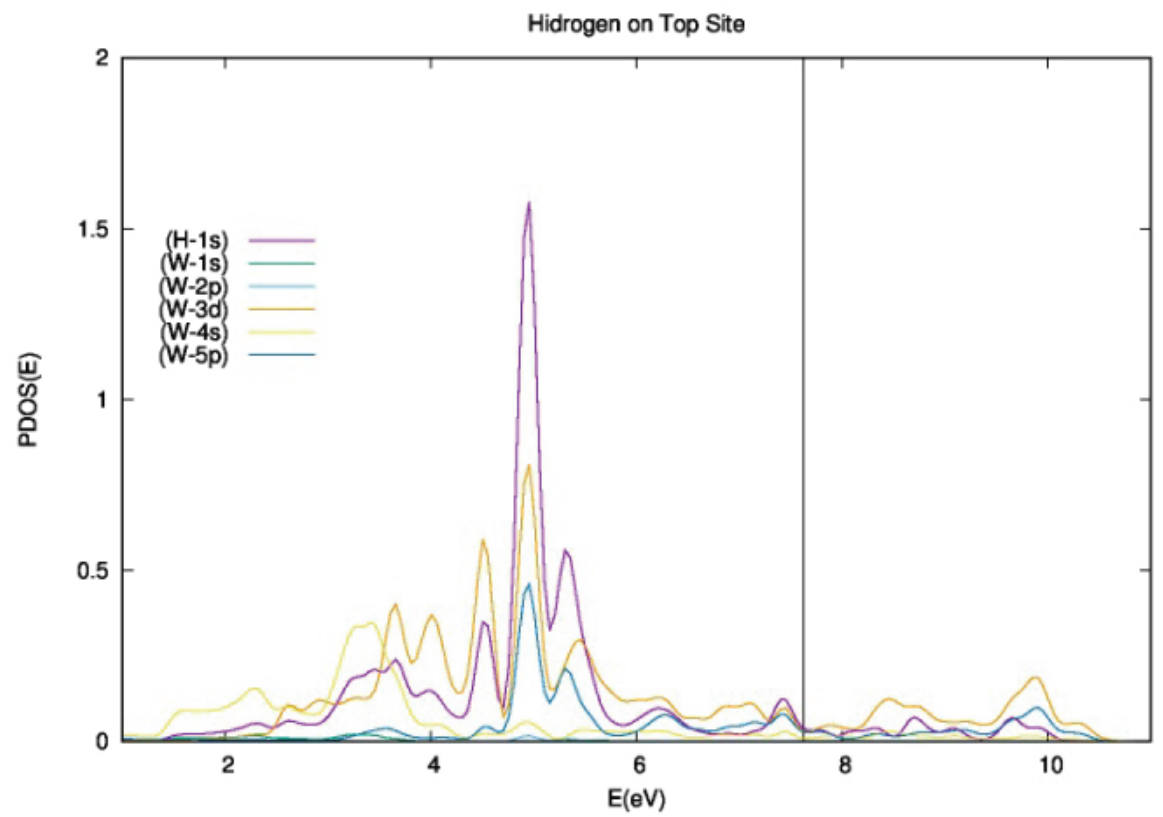

(b)

Fig. 4 (a) DOS of H-W on top site; (b) PDOS of the same system. 


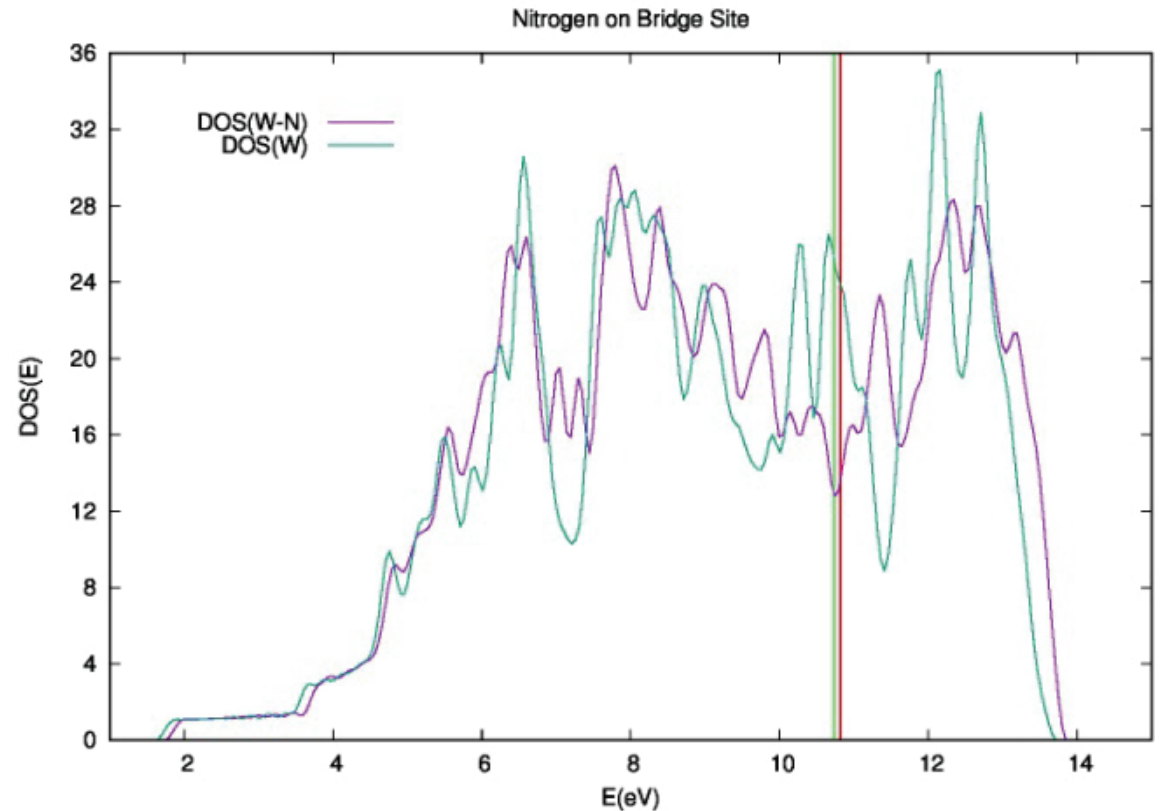

(a)

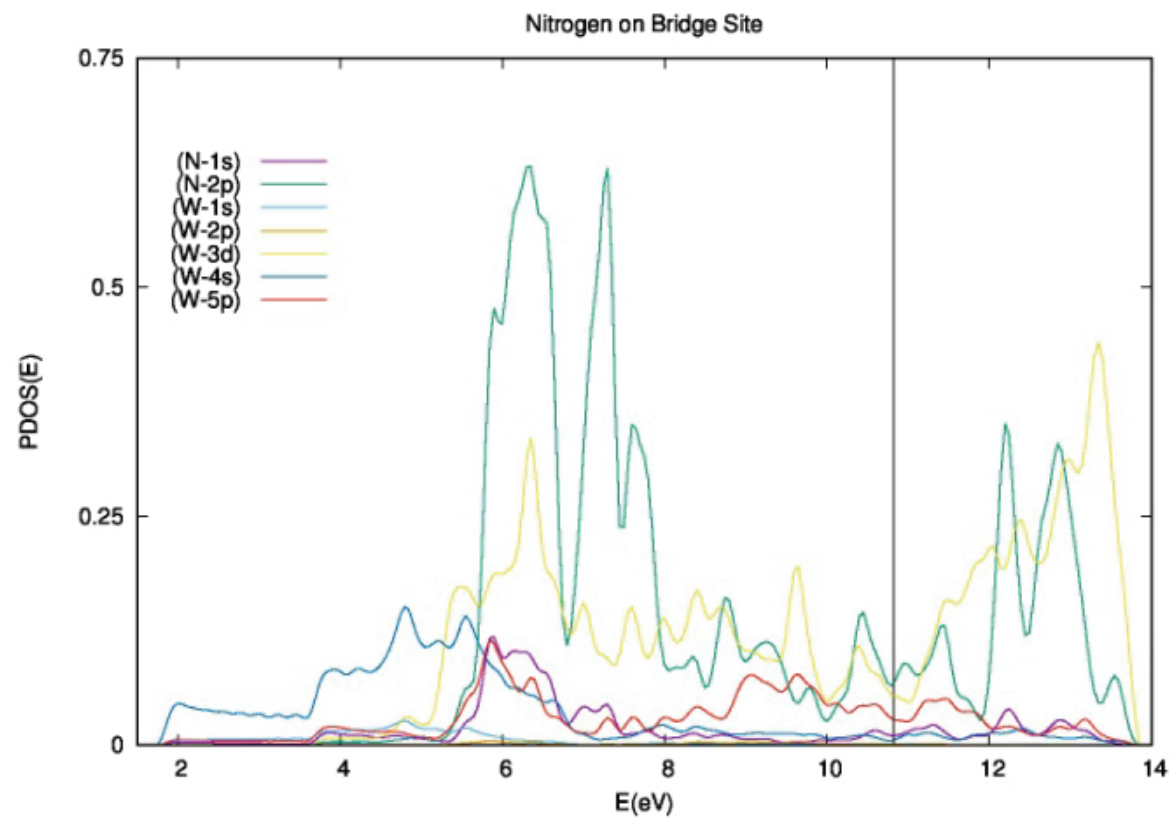

(b)

Fig. 5 (a) DOS of N-bridge site; (b) PDOS of the same system. 


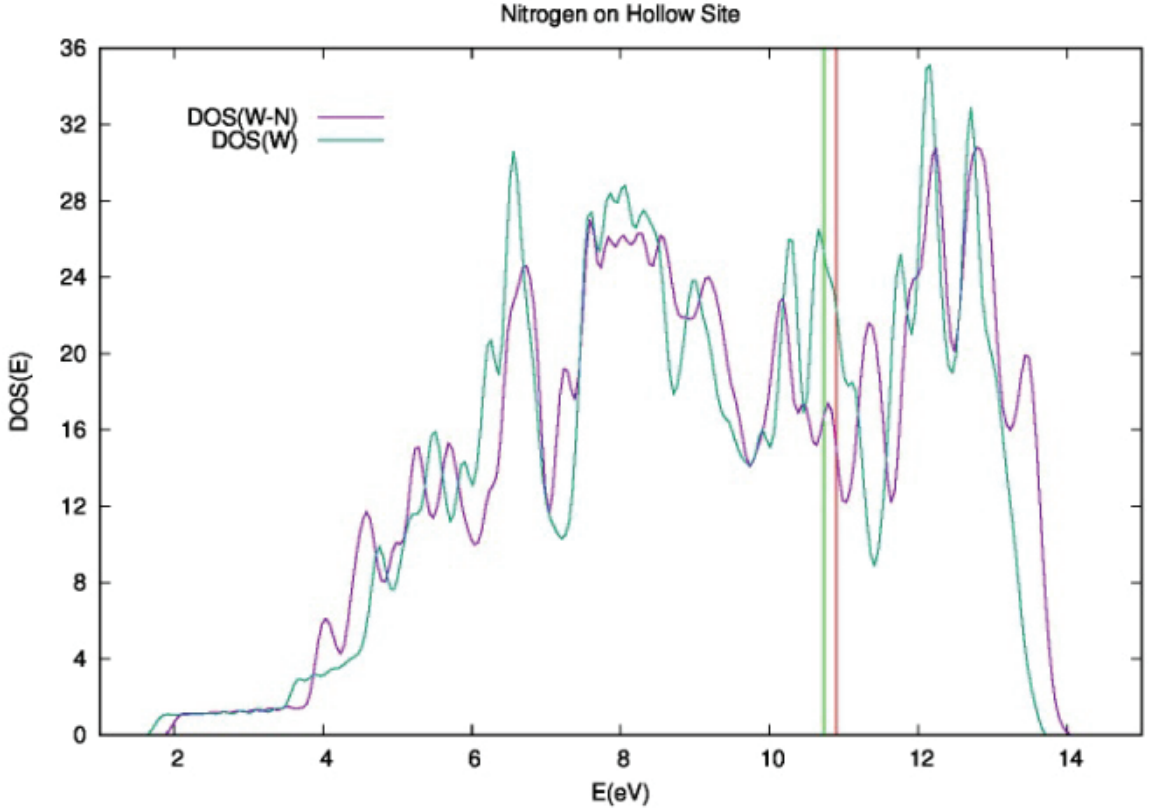

(a)

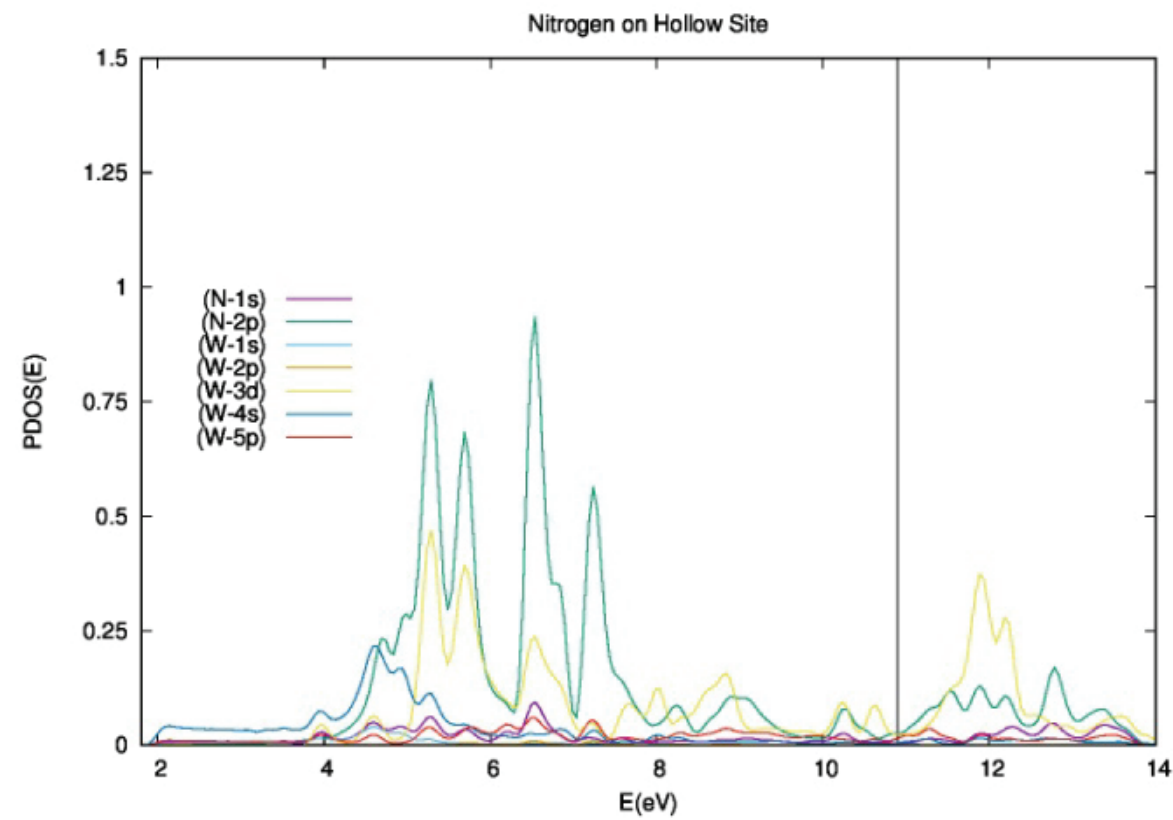

(b)

Fig. 6 (a) DOS of N-W on hollow site; (b) PDOS of the same system. 


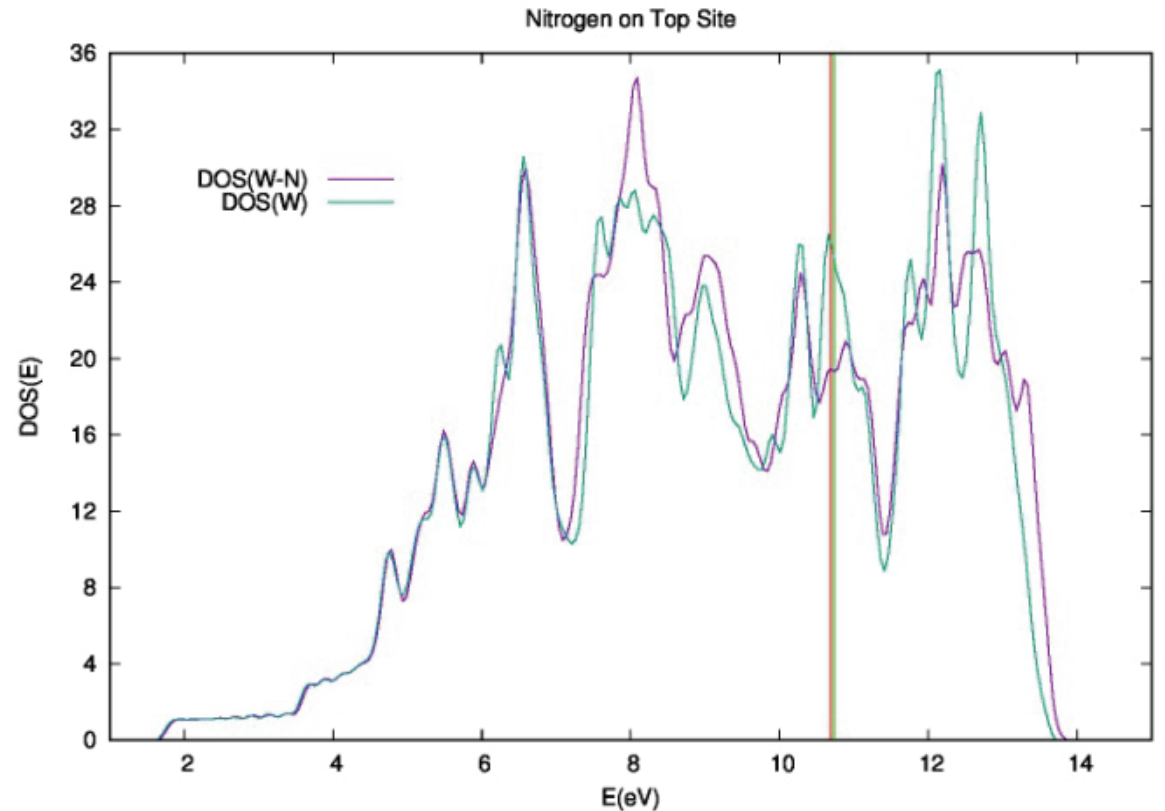

(a)

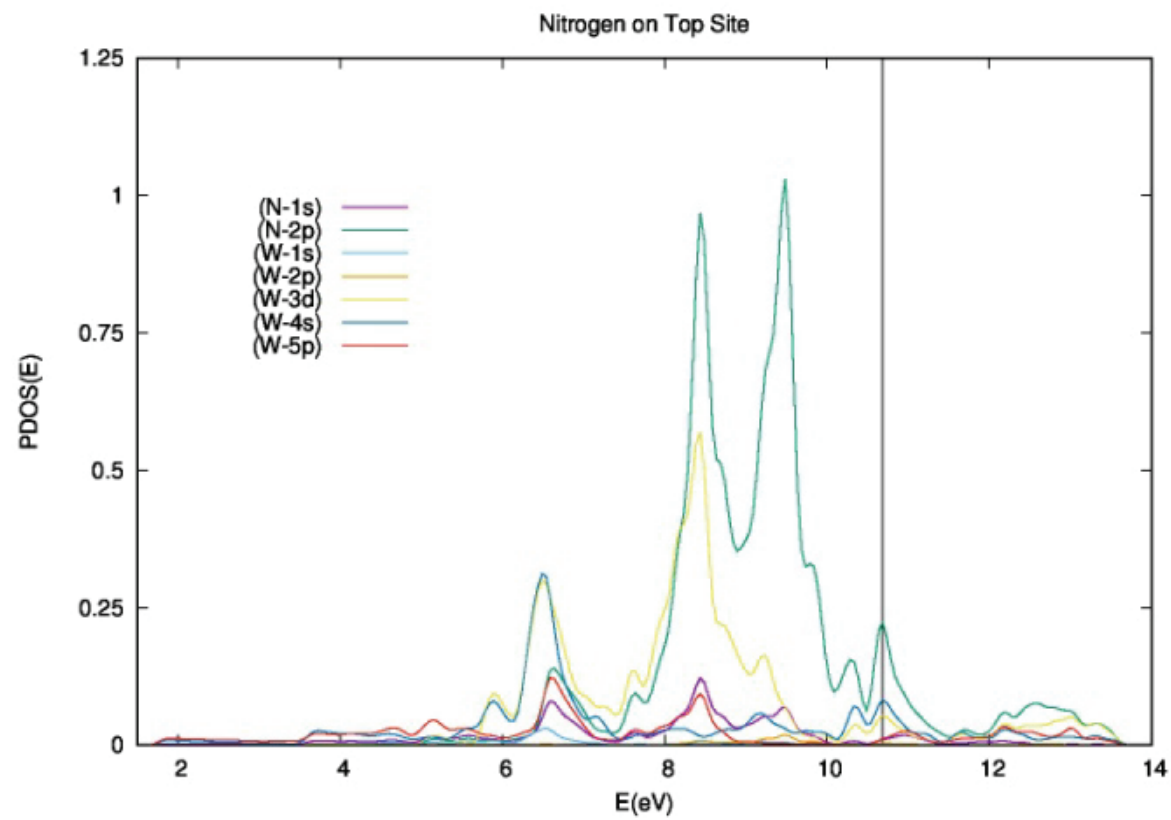

(b)

Fig. 7 (a) DOS of N-W on top site; (b) PDOS of the same system.

orbital $3 \mathrm{~d}$ for defining the conductive character of the system.

From Fig. 6a, we see that the energy $\mathrm{E}_{\mathrm{Fermi}}$ moves even more than for the interaction of the bridge site. From Fig. 6a the contribution is observed very similar near the level of the $2 p$ orbital of nitrogen and $3 d$ of the tungsten.
From Fig. 7a a lower variation of the DOS is observed near the Fermi level that is almost equal. For the projected state density (PDOS, Fig. 7a), it can be noted that the $2 p$ orbital of the nitrogen competes with the $3 \mathrm{~d}$ orbital of the tungsten to define the conductive character, but it is indisputable that the one who contributes or governs the conductive character to a 
greater extent is the orbital $2 \mathrm{~d}$ of nitrogen.

\section{Discussion and Conclusions}

In this paper, we studied the density of states of atomic $\mathrm{H}$ and $\mathrm{N}$ in $\mathrm{W}$, pointing to analyze the behavior of this system in the extreme condition of it in a fusion reactor. We used the Quantum Expresso method, applying the ultrasoft Vanderbilt pseudopotential to calculate adsorption energies of $\mathrm{H}$, as precursors of the $\mathrm{NH}_{3}$ molecule on the tungsten surface in the three sites: hollow, bridge and top. In order to achieve adsorption energies with a higher accuracy, one needs to go beyond the Quantum Expresso approximation with the consideration of more general kernel within the DFT formulation. But the goal of this work was to determine the process of adsorption in order to explain the existence of $\mathrm{NH}_{3}$ in the divertor. This development began with the simulation of ion implantation experiments, validated against existing laboratory experimental results [7]. The findings and challenges of developing a 3-D predictive capability for ammonia transport in a Tungsten divertor wall are under way.

\section{Acknowledgments}

The present work was partially subsidized by the National University of Cuyo (Project UNC 06/C367 and UNC 06-C345) and CNEA's Controlled Fusion Program.

\section{References}

[1] Zinkle, S. J. 2005. "Advanced Materials for Fusion Technology." Fusion Engineering and Design 74 (1-4): 31-40.

[2] Ehrlich, K. 1999. "The Development of Structural Materials for Fusion Reactors." Transactions of the Royal Society of London A 357 (1752): 595-617.

[3] Tanaka, S., Matera, R., Kalinin, G., Barabash, V., and Mohri, K. 1999. "ITER Materials R \& D Data Bank." Journal of Nuclear Materials 271-272 (May): 478-85.

[4] Kalinin, G., Barabash, V., Cardella, A., Dietz, J., Ioki, K., Matera, R., Santoro, R. T., and Tivey, R. 2000.
"Assessment and Selection of Materials for ITER In-Vessel Components." Journal of Nuclear Materials 283-287 (1): 10-9.

[5] Bloom, E. E., Busby, J. T., Duty, C. E., Maziasz, P. J., McGreevy, T. E., Nelson, B. E., Pint, B. A., Tortorelli, P. F., and Zinkle, S. J. 2007. "Critical Questions in Materials Science and Engineering for Successful Development of Fusion Power." Journal of Nuclear Materials 367-370 (A): $1-10$.

[6] https://www.iter.org/.

[7] Drenik, A., Vesel, A., and Mozetic, M. 2006. "Measurements of Probability for Heterogeneous Recombination of Hydrogen Atoms on Surfaces of Fusion Relevant Materials." 33rd EPS Conference on Plasma Phys. Rome, 19-23 June 2006 ECA Vol.30I, P-5.170.

[8] Decreton, M., Shikama, T., and Hodgson, E. R. 2004. "Performance of Functional Materials and Components in a Fusion Reactor." Journal of Nuclear Materials 329-333 (4): 125-32.

[9] Kaiser, H. 1881. "Condensation of Gases on Free Surfaces." Wield. Ann. 14: 451.

[10] Kohn, W., and Sham, L. J. 1965. "Self-Consistent Equations Including Exchange and Correlation Effects." Physical Review 140: A1133-8.

[11] Hohenberg, P., and Kohn, W. 1964. "Inhomogeneous Electron Gas." Physical Review B 136 (3): 864-71.

[12] Stott, M. J., and Zaremba, E. 1980. "Linear-Response Theory within the Density-Functional Formalism: Application to Atomic Polarizabilities." Physical Review A 21 (1): 12-23.

[13] Perdew, J. P., Burke, K., and Ernzerhof, M. 1996. "Generalized Gradient Approximation Made Simple." Physical Review Letters 77 (18): 3865-8.

[14] Gervasoni, J. L., and Furnari, J. C. 2014. "Density of Electron States and Volume of Solution of Hydrogen in SiC Alloys." Journal of Energy and Power Engineering 8 (2): 390-3.

[15] http://www.quantum-espresso.org/.

[16] Shikama, T., Tsuchiya, B., and Hodgson, E. R. 2007. "Effects of Neutron Irradiation on the Properties of Functional Materials for Fusion Applications: Role of Hydrogen in Radiation Effects on Oxide Ceramics." Journal of Nuclear Materials 367-370: 995-1002.

[17] Van Helden, J. H., Wagemans, W., Yagci, G., Zijlmans, R. A. B., and Schram, D. C. 2007. "Detailed Study of the Plasma-Activated Catalytic Generation of Ammonia in $\mathrm{N}_{2}-\mathrm{H}_{2}$ Plasmas" Journal of Applied Physics 101 (4): 043305 . 